\title{
Arctic Sea Ice Characterization Using RISAT-1 Compact-Pol SAR Imagery and Feature Evaluation: A Case Study Over Northeast Greenland
}

\author{
Suman Singha, Member, IEEE, and Rudolf Ressel
}

\begin{abstract}
Synthetic Aperture Radar (SAR) polarimetry has become a valuable tool in space-borne SAR-based sea ice analysis. The two major objectives in SAR-based remote sensing of sea ice are, on the one hand, to have a large coverage and, on the other hand, to obtain a radar response that carries as much information as possible in order to characterize sea ice. Single-polarimetric acquisitions of existing sensors offer a wide coverage on the ground, whereas dual polarimetric or even better fully polarimetric data offer a higher information content, which allows for a more reliable automated sea ice analysis at a cost of smaller swath. In order to reconcile the advantages of fully polarimetric acquisitions with the higher ground coverage of acquisitions with fewer polarimetric channels, hybrid/compact polarimetric acquisitions offer an excellent tradeoff between the mentioned objectives. With the advent of the RISAT-1 satellite platform, we are able to explore the potential of compact dual pol acquisitions for sea ice analysis and classification. Our algorithmic approach for an automated sea ice classification consist of two steps. In the first step, we perform a feature extraction followed by a feature evaluation procedure. The resulting feature vectors are then ingested into a trained artificial neural network classifier to arrive at a pixel-wise supervised classification. We present a comprehensive polarimetric feature analysis and classification results on a dataset acquired off the eastern Greenland coast, along with comparisons of results obtained from near-coincident (spatially and temporally) $C$-band fully polarimetric imagery acquired by RADARSAT -2 .
\end{abstract}

Index Terms-Artificial neural network, compact polarimetry (CP), feature extraction, RISAT-1, RADARSAT-2, sea ice classification.

\section{INTRODUCTION}

$\mathbf{O}$ PERATIONAL space-borne Synthetic Aperture Radar (SAR) data have become a powerful means for sea ice monitoring since the launch of the first space-borne platforms some decades ago (see, e.g., [1]-[4]). A common approach in automated sea ice classification based on SAR imagery is to first compute a vector of features (pixel-wise or using a sliding

Manuscript received November 24, 2016; revised March 1, 2017; accepted March 28, 2017. RADARSAT-2 data were accessed in the framework of Echtzeitdienste für die Maritime Sicherheit (EMS) project, funded by Federal Ministry of Education and Research (BMBF), Germany. (Corresponding author: Suman Singha.)

The authors are with the Maritime Safety and Security Laboratory, Remote Sensing Technology Institute, German Aerospace Center (DLR), Bremen 28199, Germany (e-mail: Suman.Singha@dlr.de; rudolf.ressel@ dlr.de).

Color versions of one or more of the figures in this paper are available online at http://ieeexplore.ieee.org.

Digital Object Identifier 10.1109/JSTARS.2017.2691258 window) followed by a supervised or unsupervised classification. While single-pol data can only be analyzed by classical image analysis for one channel (e.g., texture analysis), dual or fully polarimetric data allow the application of polarimetric analysis for sea ice characterization. Previous evaluations of polarimetric SAR data for sea ice characterization have shown enhanced discrimination between sea ice types and open water in both dual polarimetric (see [4]-[6]) and fully polarimetric mode (see [7] and [8]). After the polarimetric feature extraction, one then has to choose a supervised or unsupervised classification, or a segmentation approach. One of the major benefits of using Quad-Pol SAR imaging is its higher information content when interpreting different types of sea ice conditions within the SAR scenes, which facilitates us to produce improved and accurate sea ice charts. However, the disadvantage associated with fully polarimetric mode is the small spatial coverage (approximately $25-50 \mathrm{~km}$ in range direction), which restricts its use in operational ice charting services. Compact polarimetry (CP) SAR mode is capable of providing an excellent tradeoff between spatial coverage and polarimetric information content and is expected to be "as good as" fully polarimetric data, to first order [9]. One popular implementation of CP is obtained by receiving orthogonal linear polarizations when transmitting circular (left or right) polarization. The resulting hybrid-polarity (CL) architecture [10] preserves all characteristics of an end-to-end circularly polarized system while leading to a simpler radar hardware specification and more robust than end-to-end circularly polarized or end-to-end linearly polarized system [9]. Different research communities recently investigated potential applications of $\mathrm{CP}$ through simulated data derived from Quad-Pol, such as target detection in high sea state conditions [11], as well as sea ice characterization [12], [13]. The majority have concluded that the CP mode is as useful as Quad-Pol mode in terms of information content. RISAT-1 is currently the only space-borne SAR sensor with wide swath (ScanSAR) circular polarimetric modes (Tx: right circular, Rx: linear $\mathrm{H}$ and $\mathrm{V}$, hereafter addressed with $\mathrm{CL}$ Pol, [10]) and also capable of providing up to $1 \mathrm{~m}$ (Azimuth) $\times 0.67 \mathrm{~m}$ (Range) resolution over $10 \mathrm{~km} \times 10 \mathrm{~km}$ region in high-resolution mode. RISAT- 1 is also capable to operate at incidence angles as low as $12^{\circ}$. Further details of RISAT-1, different imaging and polarization modes can be found in Table I. Diverse polarimetric capabilities of RISAT-1 enable us to 
TABLE I

RISAT-1 IMAGING MODES

\begin{tabular}{|c|c|c|c|c|}
\hline Swath Coverage & \multicolumn{4}{|c|}{ Selectable within 107 to $659 \mathrm{~km}$ off-nadir (Left/Right) } \\
\hline $\begin{array}{l}\text { Incidence angle } \\
\text { coverage }\end{array}$ & \multicolumn{4}{|c|}{$12^{\circ}$ to $55^{\circ}$} \\
\hline Operating Mode & \multicolumn{4}{|c|}{ Polarization Mode } \\
\hline & $\begin{array}{l}\text { Single Pol } \\
\mathrm{HH} / \mathrm{HV} / \\
\mathrm{VH} / \mathrm{VV}\end{array}$ & $\begin{array}{c}\text { Dual Pol } \\
\mathrm{HH}+\mathrm{HV} / \\
\mathrm{VV}+\mathrm{VH}\end{array}$ & $\begin{array}{c}\text { Circular (Hybrid) Pol } \\
\text { TX: CL } \\
\text { RX: V and H (Experimental) }\end{array}$ & $\begin{array}{c}\text { Quad Pol } \\
\mathrm{HH}+\mathrm{HV}+\mathrm{VH}+\mathrm{VV}\end{array}$ \\
\hline HRS & \multicolumn{3}{|c|}{$\begin{array}{c}1 \mathrm{~m}(\text { Az. }) \times 0.67 \mathrm{~m}(\mathrm{Rz} .) \text { resol. } \\
10 \mathrm{~km} \times 10 \mathrm{~km}(10 \mathrm{~km} \times 100 \mathrm{~km} \text { Experimental }) \text { Spot } \\
\text { Min } \sigma^{0}=-16 \mathrm{~dB}\end{array}$} & \\
\hline FRS-1 & \multicolumn{3}{|c|}{$\begin{array}{l}3 \mathrm{~m}(\text { Az. }) \times 2 \mathrm{~m}(\mathrm{Rz} .) \text { resol., } \\
25 \mathrm{~km} \text { swath, Min } \sigma^{0}=-17 \mathrm{~dB}\end{array}$} & \\
\hline FRS-2 & & & $\begin{array}{l}3 \mathrm{~m}(\mathrm{Az} .) \times 4 \mathrm{~m}(\mathrm{Rz} .) \\
\text { resol., } 25 \mathrm{~km} \text { swath } \\
\text { Min } \sigma^{0}=-20 \mathrm{~dB}\end{array}$ & $\begin{array}{l}9 \mathrm{~m}(\mathrm{Az} .) \times 4 \mathrm{~m}(\mathrm{Rz} .) \\
\text { resol., } 25 \mathrm{~km} \text { swath } \\
\text { Min } \sigma^{0}=-19 \mathrm{~dB}\end{array}$ \\
\hline MRS & \multicolumn{3}{|c|}{$\begin{array}{l}21-23 \mathrm{~m}(\mathrm{Az} .) \times 8 \mathrm{~m}(\mathrm{Rz} .) \text { resol., } \\
115 \mathrm{~km} \text { swath, Min } \sigma^{0}=-17 \mathrm{~dB}\end{array}$} & \\
\hline CRS & \multicolumn{3}{|c|}{$\begin{array}{l}\text { 41-55m (Az.) } \times 8 \mathrm{~m}(\text { Rz. }) \text { resol., } \\
223 \mathrm{~km} \text { swath, Min } \sigma^{0}=-17 \mathrm{~dB}\end{array}$} & \\
\hline
\end{tabular}

TABLE II

RISAT-1 AND RADARSAT-2 IMAGING MODES USED IN THIS STUDY

\begin{tabular}{lcccccc}
\hline \hline $\begin{array}{l}\text { Date, } \\
\text { Acq. Start Time }\end{array}$ & Sensor & $\begin{array}{c}\text { Mid Inc. } \\
\text { Ang. }\end{array}$ & $\begin{array}{c}\text { Polar. } \\
\text { Mode }\end{array}$ & $\begin{array}{c}\text { Imaging } \\
\text { Mode }\end{array}$ & $\begin{array}{c}\text { Azimuth/Slant } \\
\text { Rng. Res.(m) }\end{array}$ & $\begin{array}{c}\text { Footpr. Az. } \\
/ \text { Rg. }\end{array}$ \\
\hline $2015 / 09 / 06,18: 13: 26$ & RISAT-1 & $46.63^{\circ}$ & CL & FRS-1 & $3.33 \mathrm{~m} / 2.34 \mathrm{~m}$ & $32 \mathrm{~km} / 28 \mathrm{~km}$ \\
$2015 / 09 / 06,18: 13: 30$ & RISAT-1 & $46.63^{\circ}$ & CL & FRS-1 & $3.33 \mathrm{~m} / 2.34 \mathrm{~m}$ & $32 \mathrm{~km} / 28 \mathrm{~km}$ \\
$2015 / 09 / 06,16: 38: 29$ & RISAT-1 & $27.21^{\circ}$ & CL & FRS-1 & $3.33 \mathrm{~m} / 2.34 \mathrm{~m}$ & $32 \mathrm{~km} / 25 \mathrm{~km}$ \\
$2015 / 09 / 06,16: 55: 23$ & RADARSAT-2 & $33.27^{\circ}$ & Q & FQ & $7.6 \mathrm{~m} / 5.2 \mathrm{~m}$ & $30 \mathrm{~km} / 27 \mathrm{~km}$ \\
\hline \hline
\end{tabular}

FRS-1—Fine Resolution StripMap Mode-1, FQ-FineQuad, Q- Quad Polarization (HH/HV/VH/VV), CL_Circular (Hybrid) Polarimetry Tx: Right Circular Rx: H and V

develop operational applications where polarimetric information is proven to be useful. Moreover, its both left and right looking capability and "ScanSAR" type acquisitions in CL-Pol mode makes it most suitable for operational use. Furthermore, upcoming SAR sensors, such as RADARSAT Constellation Mission (RCM), expected to be launched in 2019, with CP and larger swath coverage up to $500 \mathrm{~km}$, will change the paradigm of operational SAR-based operational sea ice characterization in near future [14]-[16].

One of our main goals is to achieve a maximal degree of automation in the process chain and provide a near real-time product; therefore, we adopt a supervised classification technique so that we can incorporate expert knowledge in our process chain. More precisely, fast artificial neural network library (FANN) (see [17]) is built into our algorithm in order to discriminate different types of sea ice. For operational purposes, the classifier needs to take into account variations due to region, season, and in particular for different incidence angle ranges, when training according to expert information (in situ observations, official ice charts). SAR images, where ice classes are known with reasonable certainty, serve as training samples so the pertaining feature information from these images can be used to train the classifier function to generate the reference output. Further details of such a neural network approach can be found in [1], in [2] for ScanSAR type imagery, in [4] for dual polarimetric $X$-band SAR, and in [8] for fully polarimetric SAR data. For the details of the FANN tools, one may confer [17]. In our paper, we apply our neural-network-based classification technique on polarimetric features extracted from RISAT-1 hybrid (CL-Pol) mode imagery of sea ice. As mentioned before, the RISAT-1 platform carries the first space-borne SAR sensor that is capable of acquiring CL-Pol data in different imaging modes, which gives us the unique opportunity to assess the capability of this polarimetric mode for operational exploitation. Theoretical details for hybrid dual polarimetry can be found in [18] and [19] and the literature references therein. Recent studies by [12] and [20] provide a survey of these features on simulated compact pol data derived from RADARSAT-2 Quad-Pol data. The references for the particular polarimetric parameters used in this study are given in Section III.

This paper is organized as follows: Section II provides the description of the RISAT- 1 and RADARSAT- 2 dataset used in this study. Section III provides the summary of the proposed methodology and the processing chain along with a scheme to analyze the relevancy of polarimetric. Experimental results and discussions about the performance of the classifier and feature 


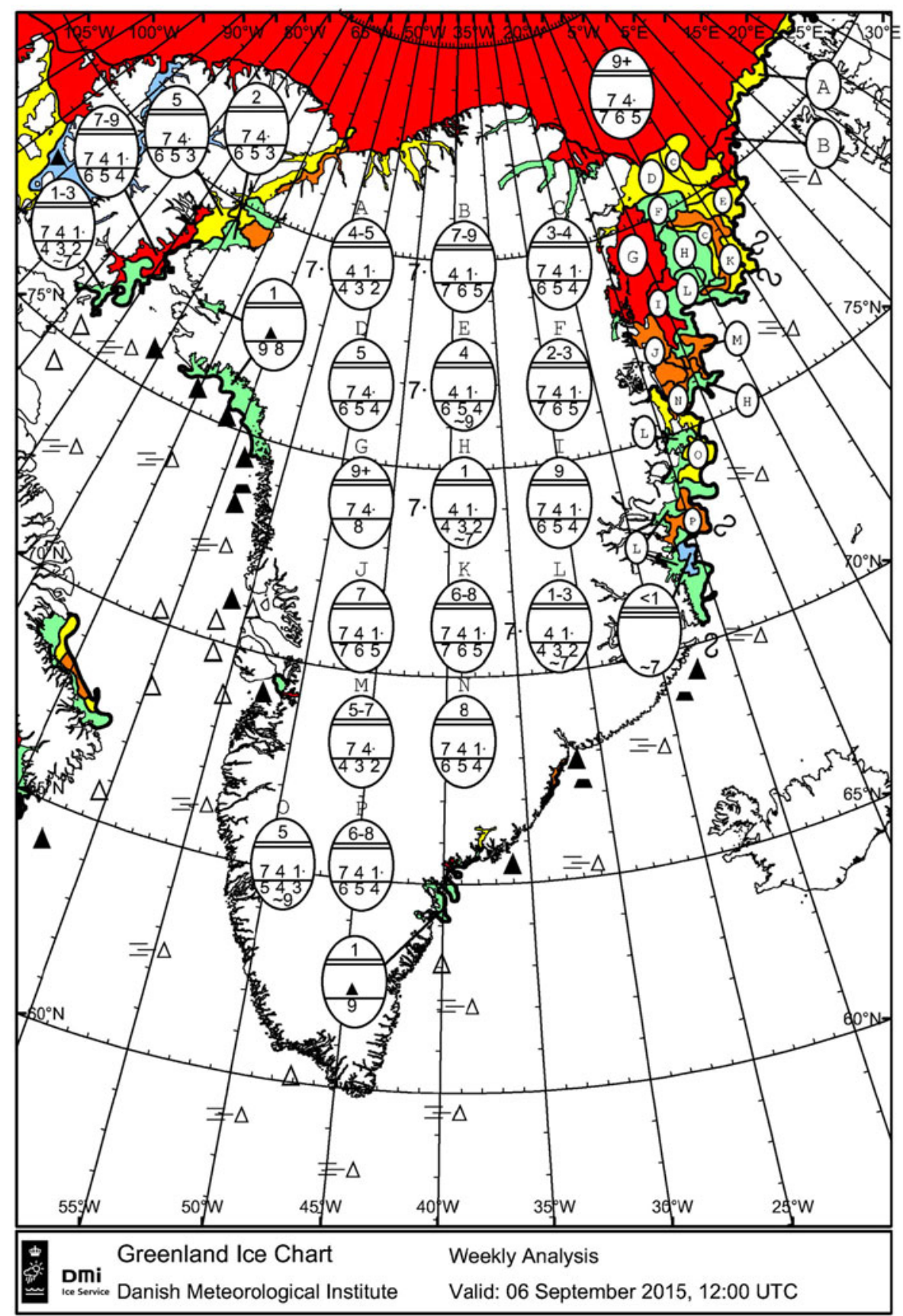

Fig. 1. Greenland Ice Chart Weekly analysis on September 6, 2015 (provided by DMI ice service).

analysis are presented in Section IV, while summary and conclusions are drawn in Section V.

\section{DATASET}

The dataset we used to develop the proposed algorithm were two adjacent and overlapping acquisitions by the RISAT- 1 satellite. Acquisition date was 2015/09/06, 18:13 (UTC), center locations of the two image frames are $78.14 \mathrm{~N}, 17.05 \mathrm{~W}$, and $77.89 \mathrm{~N}, 16.72 \mathrm{~W}$, off the Northeastern Greenland coast over the Atlantic. The image mode was Fine Resolution StripMap
(FRS-1) with a resolution of $3 \mathrm{~m}$ in azimuth and $2 \mathrm{~m}$ in range and a swath width of $25 \mathrm{~km}$. The transmitted $C$-band beam was right circular polarized $(\mathrm{R})$, the receiving linear polarization were vertical $(\mathrm{V})$ and horizontal $(\mathrm{H})$, a mode uniquely available in RISAT-1. Besides these circular-transmit/linear-receive modes (CL-Pol), the FRS-1 StripMap mode, as well as the ScanSAR modes, can also be obtained in linear single-pol modes and dual-pol (HH-HV, VV-VH) mode. There is also a provision of obtaining fully polarimetric image with slightly lower resolution StripMap mode (FRS-2). Fig. 2 shows the regional high-resolution sea ice chart over eastern Greenland region, 


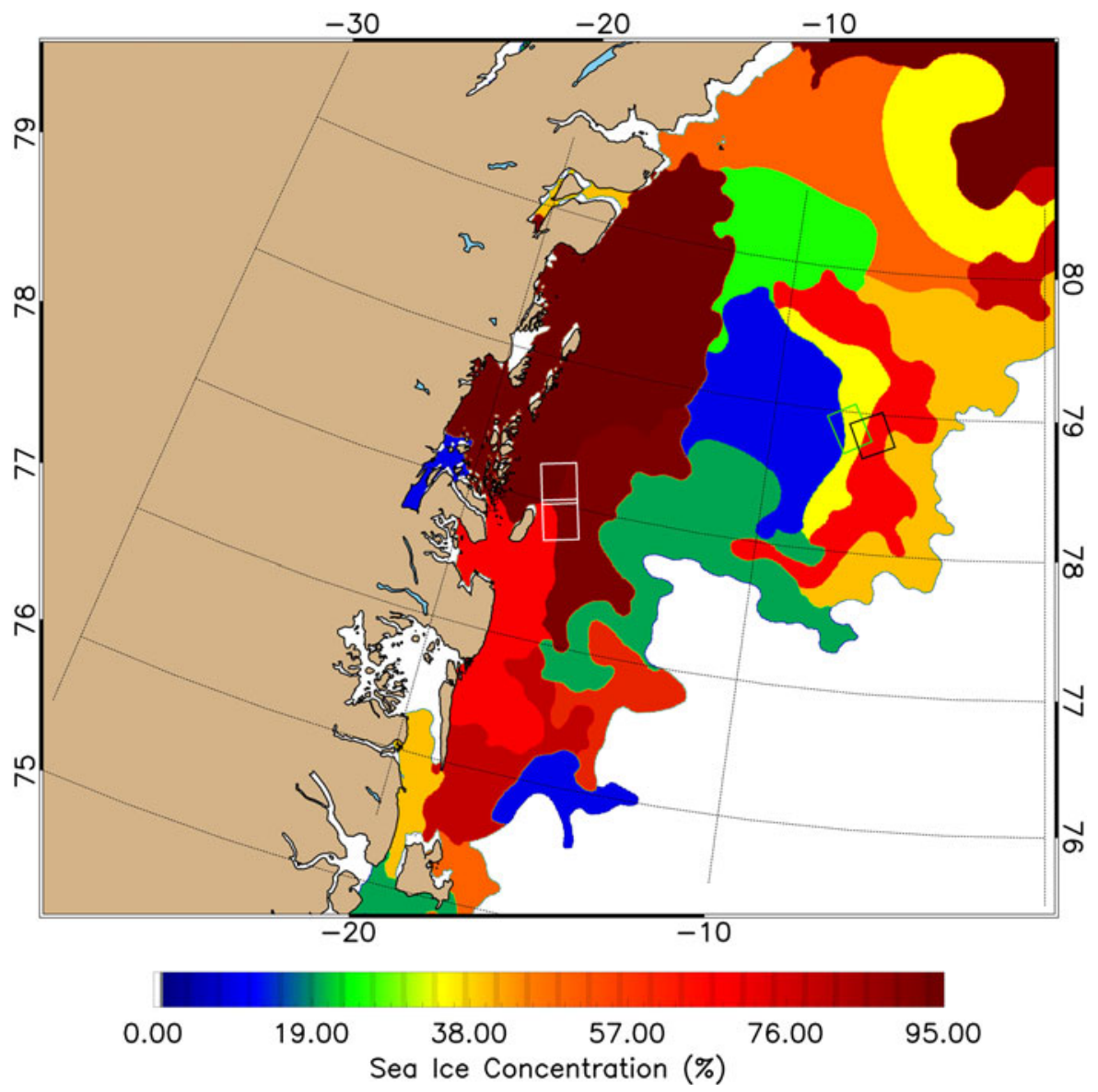

Fig. 2. Sea ice concentration on September 6, 2015 (provided by DMI). Rectangles indicate the footprint of the RISAT-1 and RADARSAT-2 acquisitions. The left image pair (in white rectangles) was acquired by RISAT-1 on September 6, 2015, at 18:13 UTC. The right image pair was acquired on September 6, 2015, by RISAT-1 at 16:38 UTC (green rectangle) and RADARSAT-2 at 16:55 (black rectangle) with temporal distance of approximately 17 min.

produced on September 6, 2015, at 12:00 UTC. The operational ice chart (ice concentration) was obtained through Copernicus Marine Environment Monitoring Service (CMEMS) and produced by danish meteorological institute (DMI). The relationship between sea ice concentration and sea ice types can be found in [21]. This ice chart was used to help identifying the ice types in the RISAT-1 SAR scene. In case of unavailability of in situ data, operational ice charts are considered the most reliable source of information when characterizing sea ice in the Arctic. It is evident from Figs. 1 (Egg Code : G, I, and J) and 2 that a very high concentration of ice $(60-90 \%)$ prevails in case of RISAT-1 acquisition of 18:13 UTC (shown in white rectangles). The dominant ice types we identified were open water/nilas (OW), young ice (YI), smooth first year ice (SFYI), and rough first/multiyear ice (RFMYI), where the degree of roughness is to be understood as visually perceivable deformation (Figs. 4 and 6). On the other hand, very low ice concentration (mainly open waters) was reported for RISAT-1 acquisition of 16:38 UTC (shown in green rectangle in Fig. 2). It is also noticeable from the ice concentration map that the RADARSAT-2 acquisition of 16:25 UTC covers two different ice regime.

In order to validate the proposed methodology with an established methodology based on Quad-Pol [8], we obtained a near coincident (spatially and temporally) RISAT-1 FRS-1 (CL-Pol) and RADARSAT-2 Fine Quad-Pol imagery off the Northeastern Greenland. The RISAT-1 imagery was acquired on September 6 at 16:38 (UTC) and overlapping RADARSAT-2 imagery was acquired on the same day at 16:55 (UTC), i.e., $17 \mathrm{~min}$ after the RISAT acquisition. RISAT- 1 and RADARSAT-2 imageries are presented in Figs. 7 and 8 (left-hand side) where similar ice situation could be observed.

\section{Methodology}

Our algorithmic approach consists of two steps: First, we extract for each pixel a vector of 21 polarimetric features (we refer to these 21 values for each pixel as "vector"), followed by selection of training/validation patches for artificial neural network classifier. As mentioned before, due to unavailability of ground truth for the selection of training/validation patches, we relied on ancillary information from ice charts and ice concentration map by the Danish Meteorological Service (Figs. 1 and 2) for the acquisition date and also on expert visual judgment of the feature images and their $m-\chi$ RGB compositions. It is also important to note here that the training/validation patches were selected in such a way that it adequately represent 


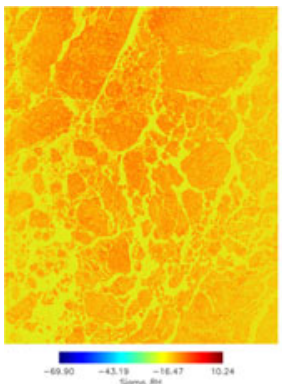

(a)

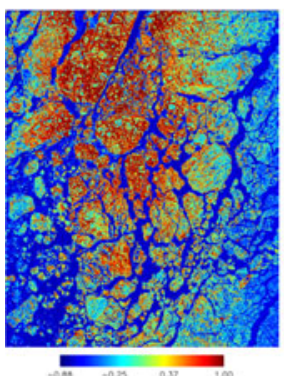

(g)

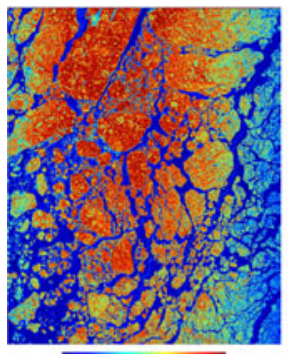

$(\mathrm{m})$

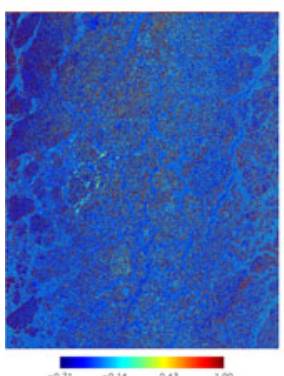

(s)

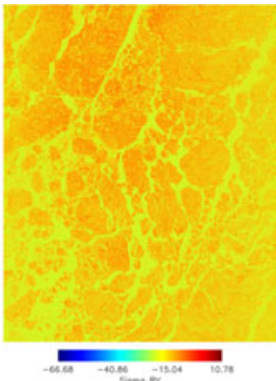

(b)

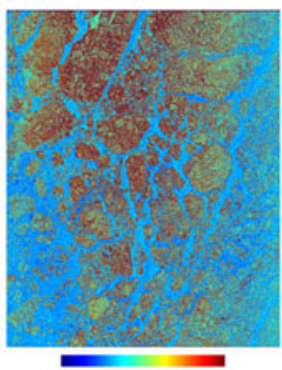

(h)

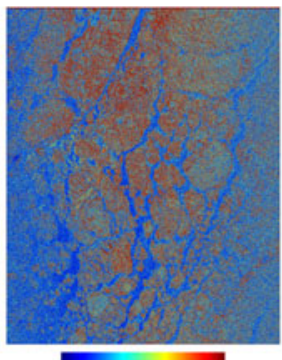

(n)

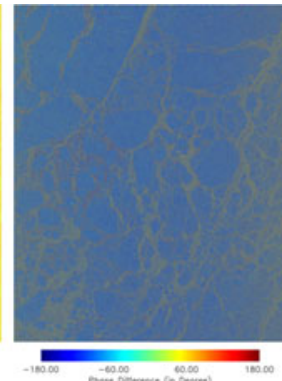

(c)

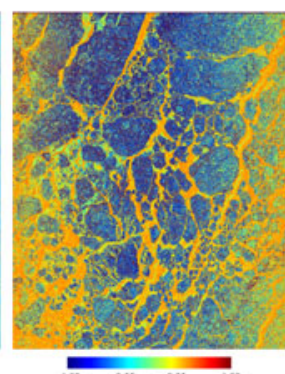

(i)

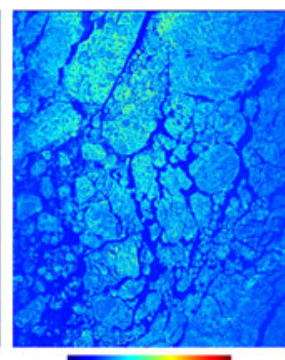

(o)

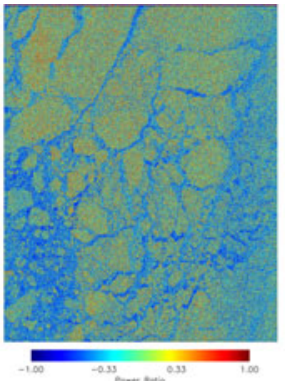

(d)

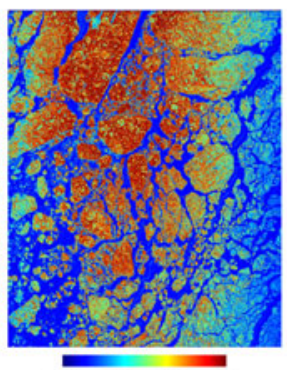

(j)

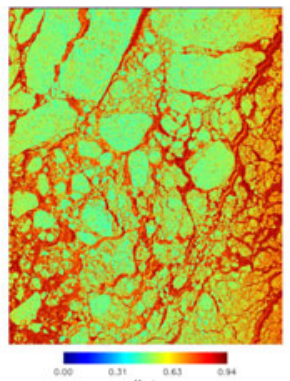

(p)

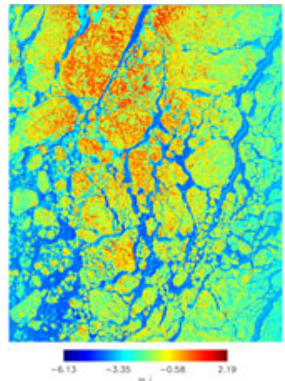

(e)

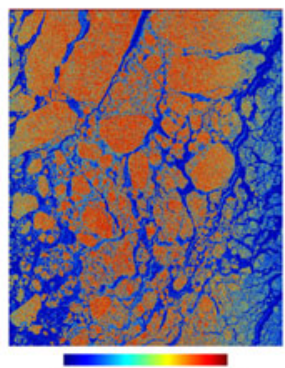

(k)

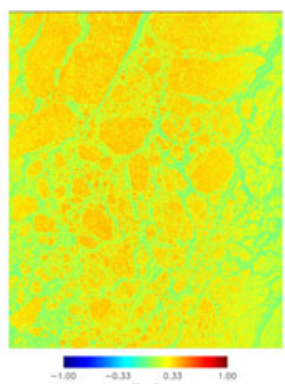

(q)

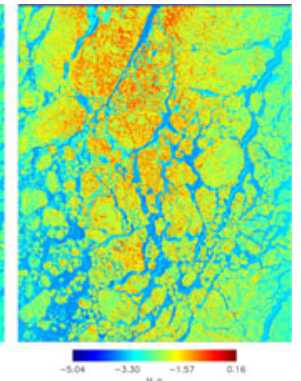

(f)

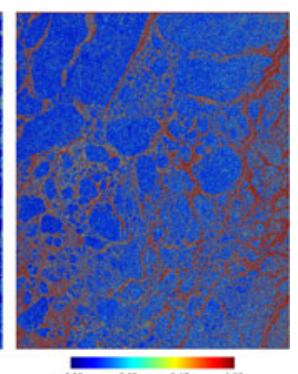

(1)

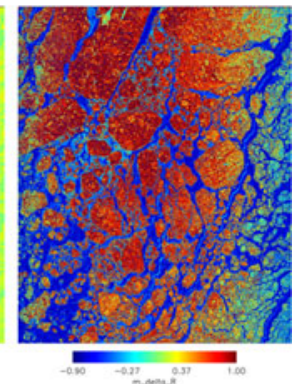

(r)

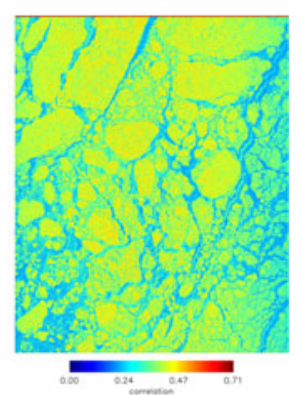

(u)

Fig. 3. Images of different polarimetric features for RISAT-1 FRS-1 acquisition presented in Fig. 4. (a) $\sigma_{\mathrm{RH}}$. (b) $\sigma_{\mathrm{RV}}$. (c) $\delta$. (d) $\gamma$. (e) $H_{i}$. (f) $H_{p}$. (g) $S_{1}$. (h) $S_{2}$. (i) $S_{3}$. (j) $S_{4}$. (k) $m$. (l) $\sin 2 \chi$. (m) $m-\chi_{B}$. (n) $m-\chi_{R}$. (o) $m-\chi_{G}$. (p) $\mu_{c}$. (q) $\mu_{E}$. (r) $V_{R}$. (s) $V_{B}$. (t) $V_{G}$. (u) $\rho$.

the incidence angle variation. Then, we feed each such vector into a neural network classifier for training and validation purpose [8].

When the polarimetric parameters are derived from the covariance or coherency matrix, we used an $11 \times 11$ pixel sliding window for averaging purposes. The features/polarimetric parameters were extracted from the full resolution single look complex image layers $(\mathrm{RH}, \mathrm{RV})$, with about $11300 \times 13900$ pixels per layer. The polarimetric parameters were taken from existing research in [9], [12], [18], and [22]. The basis of our analysis are the complex valued image layers $\left(S_{\mathrm{RH}}, S_{\mathrm{RV}}\right)$, where we define the notation

$$
S_{\mathrm{RH}}=\sigma_{\mathrm{RH}} \exp \left(j \varphi_{\mathrm{RH}}\right) .
$$

Resulting features are the intensities

$$
\sigma_{\mathrm{RH}}, \sigma_{\mathrm{RV}}
$$

the phase difference (see [9])

$$
\delta=\varphi_{\mathrm{RH}}-\varphi_{\mathrm{RV}} .
$$

It is pointed out by [6] and [20] that the responses of $\sigma_{\mathrm{RH}}$ and $\sigma_{\mathrm{RV}}$ are analogous to the conventional $\sigma_{\mathrm{HH}}$ and $\sigma_{\mathrm{VV}}$. Therefore, 

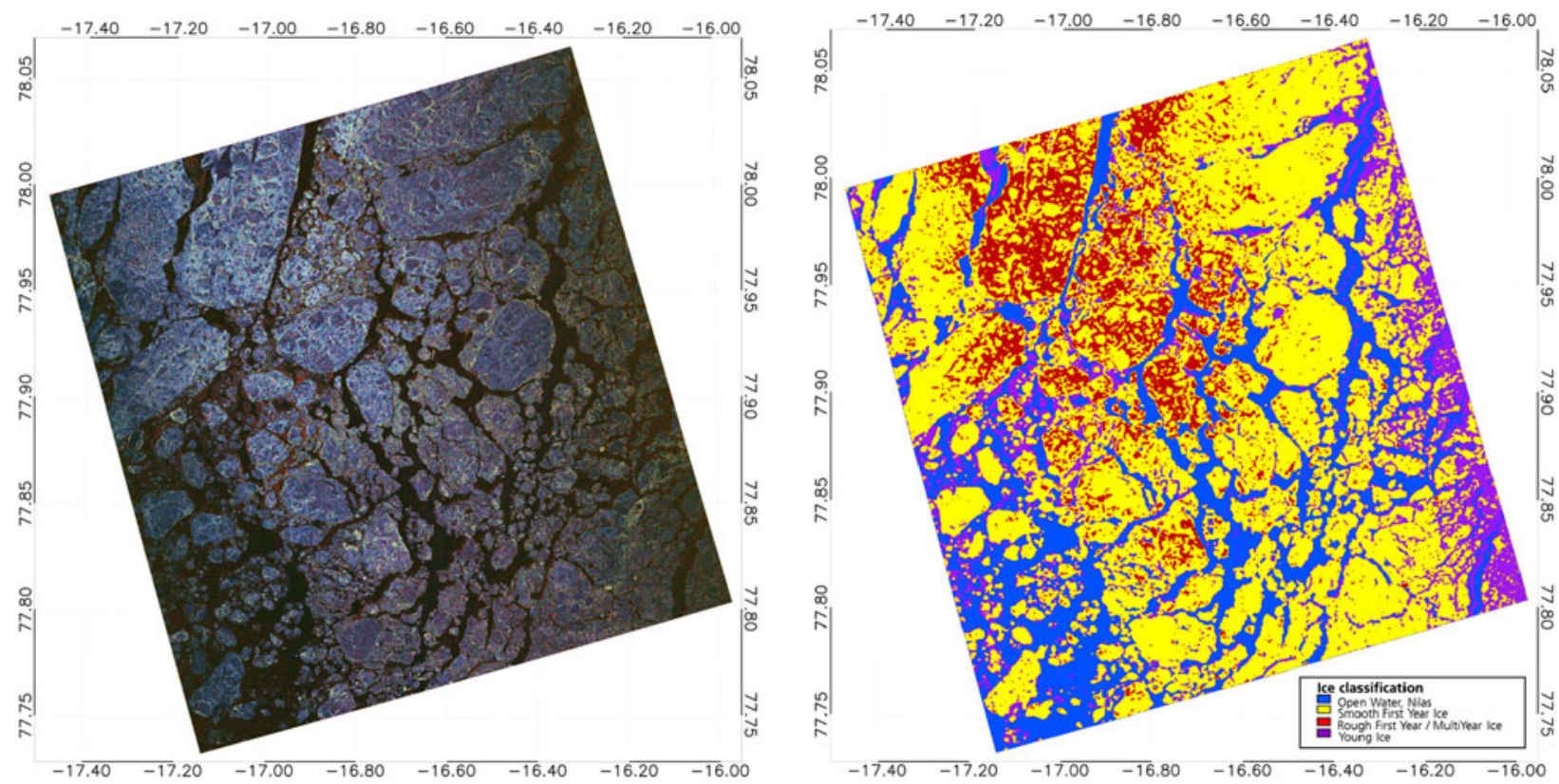

Fig. 4. Left: Geocoded $m-\chi$ RGB composite of RISAT-1 acquisition (FRS-1) on September 6, 2015, 18:13:26 UTC. Right: Classification result of RISAT-1 acquisition on September 6, 2015, 18:13:26 UTC, Open water/nilas (OW) blue, young ice (YI) purple, smooth first year ice (SFYI) yellow, rough first/multiyear ice (RFMYI) red.

their backscatter is expected to be low for the YI, SFYI and open water at low wind speeds, and higher for the RFMYI. Those studies also pointed out that $\delta$ and $\gamma$ for the CL-Pol case should also behave similarly to those for the traditional copolarized (HH, VV) case.

The circular (right) copolarized ratio

$$
\gamma=\frac{\left\langle\sigma_{\mathrm{RH}}\right\rangle}{\left\langle\sigma_{\mathrm{RV}}\right\rangle} .
$$

Raney et al. [18] define the four Stokes parameters $S_{1}, S_{2}$, $S_{3}, S_{4}$ by

$$
\begin{aligned}
& S_{1}=\left\langle\left|S_{\mathrm{RH}}\right|^{2}+\left|S_{\mathrm{RV}}\right|^{2}\right\rangle \\
& S_{2}=\left\langle\left|S_{\mathrm{RH}}\right|^{2}-\left|S_{\mathrm{RV}}\right|^{2}\right\rangle \\
& S_{3}=2 \operatorname{Re}\left\langle S_{\mathrm{RH}} S_{\mathrm{RV}}^{*}\right\rangle
\end{aligned}
$$

and

$$
S_{4}=-2 \operatorname{Im}\left\langle S_{\mathrm{RH}} S_{\mathrm{RV}}^{*}\right\rangle .
$$

From these, one derives the degree of polarization

$$
m=\sqrt{S_{2}^{2}+S_{3}^{2}+S_{4}^{2}} / S_{1} .
$$

The degree of circularity is given by

$$
\sin 2 \chi=-S_{4} /\left(m S_{1}\right)
$$

From $m$ and $\chi$, Raney et al. [18] derive the $m-\chi$ decomposition by

$$
\begin{aligned}
m-\chi_{B} & =\left[m S_{1}(1-\sin 2 \chi) / 2\right]^{1 / 2} \\
m-\chi_{R} & =\left[m S_{1}(1+\sin 2 \chi) / 2\right]^{1 / 2} \\
m-\chi_{G} & =\left[S_{1}(1-m)\right]^{1 / 2}
\end{aligned}
$$

where $\mathrm{B}$ indicates the single-bounce (Bragg) backscatter, $R$ represents double-bounce, and $G$ corresponds to randomly polarized backscatter. The work in [9], furthermore, proposes the following features: circular (right) polarization ratio

$$
\mu_{c}=\frac{S_{1}-S_{4}}{S_{1}+S_{4}}
$$

ellipticity

$$
\mu_{E}=\frac{S_{4}}{S_{1}} .
$$

With $S_{1}, \delta$, and $m$, Charbonneau et al. [9] derive the $m-\delta$ decomposition:

$$
\begin{aligned}
V_{R} & =\sqrt{S_{1} m \frac{(1-\sin \delta)}{2}} \\
V_{G} & =\sqrt{S_{1}(1-m)} \\
V_{B} & =\sqrt{S_{1} m \frac{(1+\sin \delta)}{2}}
\end{aligned}
$$

where $V_{R}$ is related to double-bounce, $V_{G}$ is related to volumetric, and $V_{B}$ is related to surface scattering. A recent publication 


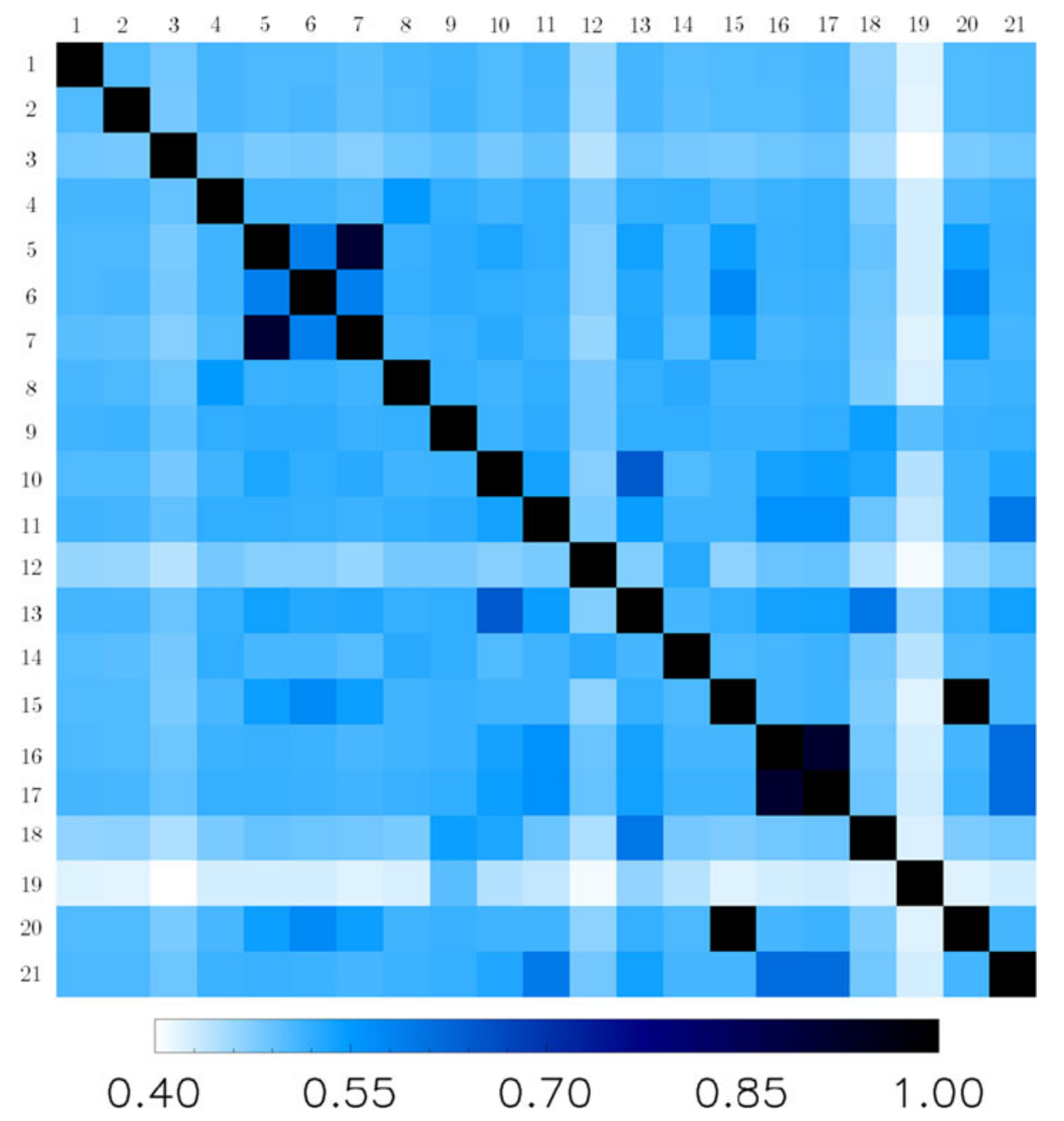

Fig. 5. Normalized mutual information for RISAT-1 image of September 6, 2015, 18:13:26 UTC (Fig. 4), $\mathcal{I}\left(Y_{1}, Y_{2}\right) / \sqrt{\mathcal{H}\left(Y_{1}\right) \mathcal{H}\left(Y_{2}\right)}$. Features are 1: $\sigma_{\mathrm{RH}}, 2$ : $\sigma_{\mathrm{RV}}, 3: \delta, 4: \gamma, 5: H_{i}, 6: H_{p}, 7: S_{1}, 8: S_{2}, 9: S_{3}, 10: S_{4}, 11: m, 12: \sin 2 \chi, 13: m-\chi_{B}, 14: m-\chi_{R}, 15: m-\chi_{G}, 16: \mu_{c}, 17: \mu_{E}, 18: V_{R}, 19: V_{B}, 20:$ $V_{G}, 21: \rho$

[12] introduced the features correlation coefficient of amplitudes

$$
\rho=\frac{\sqrt{\left|\left\langle S_{\mathrm{RH}} S_{\mathrm{RV}}^{*}\right\rangle\right|}}{\sqrt{\left|S_{\mathrm{RH}} S_{\mathrm{RH}}^{*}\right|+\left|S_{\mathrm{RV}} S_{\mathrm{RV}}^{*}\right|}} .
$$

When one defines the coherency matrix $T_{2}$ of the scattering vector $k=\left(S_{\mathrm{RH}}, S_{\mathrm{RV}}\right)^{t r}$, one can define (as is done in [12]) the Shannon entropy of intensity components

$$
H_{i}=2 \log \left(\frac{\pi e \operatorname{Tr}\left(T_{2}\right)}{2}\right)
$$

and the Shannon entropy of the polarimetric component

$$
H_{p}=\log \left(\frac{4 \operatorname{det} T_{2}}{\operatorname{Tr}\left(T_{2}\right)^{2}}\right)
$$

where $\operatorname{Tr}(\cdot)$ denotes the matrix trace and det denotes the determinant.

In order to extract above mentioned CL-Pol features from calibrated $\left(S_{\mathrm{RH}}, S_{\mathrm{RV}}\right)$, we used the interactive data language (IDL) [23]. The neural network responsible for classification was based on the FANN library, programmed in $\mathrm{C}$, which was "bridged" into our IDL implementation of the process chain. After feature extraction, the feature matrices were down-sampled by a user defined factor and rescaled to meet the requirements of the neural network input data range, resulting in an output classified image with dimension reduced by the user defined factor.

The training data consisted of small patches summing in total about 3000-4000 pixels per ice type, taken only from RISAT-1 acquisition presented in Fig. 4. Training patches were determined by visual judgment of the feature images in conjunction with DMI provided operational ice charts. In the network topology, we used two hidden layers with 14 and 16 hidden neurons, and for training of the neural network we exploited the RPROP (Resilient Backpropagation) algorithm [17]. Before ingesting in a neural network, as prerequisite all features were rescaled into the range $[-1.0,1.0]$. A common nonlinear rescaling method using the tanh function was implemented:

$$
\tilde{x}=\tanh \left(\frac{x-\bar{X}}{\nu_{X}}\right)
$$



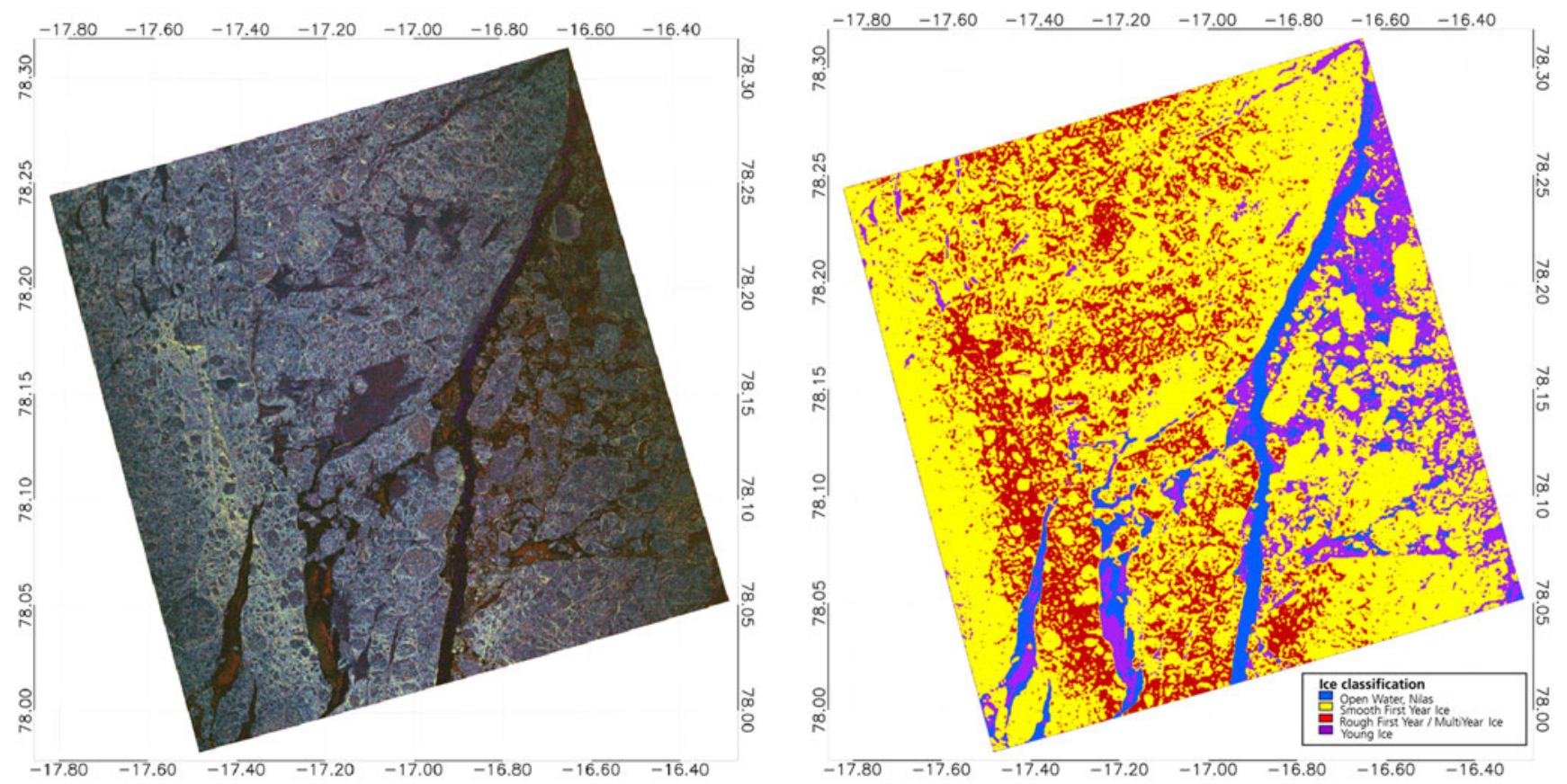

Fig. 6. Left: Geocoded $m-\chi$ RGB composite of RISAT-1 acquisition (FRS-1) on September 6, 2015, 18:13:30 UTC. Right: Classification result of RISAT-1 acquisition on September 6, 2015, 18:13:30 UTC. Open water/nilas (OW) blue, young ice (YI) purple, smooth first year ice (SFYI) yellow, rough first/multiyear ice (RFMYI) red.

TABLE III

CLASSIFICATION RESUlts COMPARED TO REFERENCE DATA SAMPLES FROM EACH CLASS, 18:13 UTC RISAT-1 ACQUISITION (FIG. 4)

\begin{tabular}{lcccc}
\hline \hline & \multicolumn{4}{c}{ Reference Ice Class } \\
\cline { 2 - 5 } ANN Classification & OW & YI & SFYI & RFYMYI \\
\hline OW & $100.0 \%$ & $\%$ & $0 \%$ & $0 \%$ \\
YI & $0 \%$ & $96.3 \%$ & $0 \%$ & $0 \%$ \\
SFYI & $0 \%$ & $1.1 \%$ & $97.2 \%$ & $3.0 \%$ \\
RFYMYI & $0 \%$ & $2.6 \%$ & $2.8 \%$ & $97.0 \%$ \\
\hline \hline
\end{tabular}

where $\bar{X}$ denotes the mean of all values of feature $X$ in the training data and $\nu_{X}$ denotes the standard deviation of all values of feature $X$ in the training data [3]. After a neural network has been trained on data rescaled with these particular training data statistical parameters $\mathcal{P}=\left\{\bar{X}, \nu_{X}\right\}$, any feature vector that is fed into this network for classification must be rescaled in the similar way with these parameters $\mathcal{P}$ before classification. The entire statistical analysis was conducted on the rescaled feature values, since they determine the behavior of the classifier. The algorithm was run on a virtual Linux machine with six logical cores (Intel Core i-7 3740 QM, 11 GB RAM) with a runtime of about $15 \mathrm{~min}$ (using a trained network) for processing an RISAT-1 scene with nominal azimuth coverage, which includes image ingestion, calibration, polarimetric feature extraction, feature rescaling, classification, and generation of classified image in Geotiff format.

As mentioned in the Section II, we have also investigated the classification results obtained from $C$-band CL-Pol imagery with the classification results obtained from $C$-band Quad-Pol imagery. Until now, no such direct comparison on
TABLE IV

RELEVANCE FOR DistingUishing All DifFERENT Classes (All-Class-Relevance) BASED ON RISAT-1, SEPTEMBER 6, 2015 (INCIDENCE ANGLE: $46.63^{\circ}$, Fig. 4)

\begin{tabular}{lcc}
\hline \hline Feature & $\backslash$ boldmath $\mathcal{I}_{0}$ & $\backslash$ boldmath $\mathcal{I}_{1}$ \\
\hline$H_{i}$ & 1.0593969 & 0.46637396 \\
$S_{1}$ & 1.0424971 & 0.46206168 \\
$H_{p}$ & 1.0166368 & 0.44744205 \\
$m-\chi_{B}$ & 0.86767807 & 0.38002773 \\
$V_{G}$ & 0.82430118 & 0.36413844 \\
$m-\chi_{G}$ & 0.82430118 & 0.36413844 \\
$V_{R}$ & 0.78547706 & 0.34672178 \\
$S_{4}$ & 0.76447945 & 0.34587210 \\
$\mu_{c}$ & 0.51335117 & 0.22496472 \\
$\mu_{E}$ & 0.51291933 & 0.22488643 \\
$m$ & 0.51196854 & 0.22422554 \\
$\rho$ & 0.47133816 & 0.20697907 \\
$S_{2}$ & 0.42400309 & 0.18628973 \\
$V_{B}$ & 0.35554936 & 0.16725667 \\
$\gamma$ & 0.35365488 & 0.15499660 \\
$S_{3}$ & 0.35177549 & 0.15371626 \\
$\sin 2 \chi$ & 0.31450092 & 0.14272602 \\
$m-\chi_{R}$ & 0.29188683 & 0.12875295 \\
$\sigma_{\mathrm{R}} \mathrm{H}$ & 0.24465867 & 0.10777630 \\
$\sigma_{\mathrm{RV}}$ & 0.22810091 & 0.10051446 \\
$\delta$ & 0.17789396 & 0.079571969 \\
\hline \hline
\end{tabular}

$\mathcal{I}_{0}$ denotes $\mathcal{I}(X \mid$ Class(all $\left.)\right)$ and $\mathcal{I}_{1}$ denotes $\mathcal{I}(X \mid$ Class $($ all $)) / \sqrt{\mathcal{H}(X)}$. Where $\sqrt{\mathcal{H}(X)}$ denotes the entropy of random variable $X$.

near-coincident, Quad-Pol, and CL-Pol space-borne SAR data acquired over the Arctic has been conducted. This temporal and spatial correlation of Quad-Pol and CL-Pol data allows us to make statements on the classification results, which can be regarded as rather unaffected by any uncertainty related to space and time variation impact. In order to classify the 
TABLE V

RISAT-1, SEPTEMBER 6, 2015 (INCIDENCE ANGLE: 46.63ํ․ Fig. 4): TwO-CLASS-RELEVANCE IN DESCENDING ORDER FOR DIFFERENT PAIRS OF CLASSES

\begin{tabular}{|c|c|c|c|c|c|}
\hline 1 versus 2 & 1 versus 3 & 1 versus 4 & 2 versus 3 & 2 versus 4 & 3 versus 4 \\
\hline$m-\chi_{G}$ & $S_{1}$ & $H_{i}$ & $m-\chi_{B}$ & $H_{p}$ & $H_{p}$ \\
\hline$H_{i}$ & $H_{p}$ & $S_{1}$ & $S_{1}$ & $H_{i}$ & $V_{G}$ \\
\hline$S_{4}$ & $H_{i}$ & $m-\chi_{B}$ & $H_{i}$ & $S_{1}$ & $m-\chi_{G}$ \\
\hline$S_{1}$ & $V_{G}$ & $S_{4}$ & $S_{4}$ & $V_{G}$ & $H_{i}$ \\
\hline$H_{p}$ & $m-\chi_{G}$ & $V_{R}$ & $V_{R}$ & $m-\chi_{G}$ & $S_{1}$ \\
\hline$V_{R}$ & $m-\chi_{B}$ & $H_{p}$ & $H_{p}$ & $m-\chi_{B}$ & $V_{R}$ \\
\hline$V_{G}$ & $\gamma$ & $V_{G}$ & $\mu_{E}$ & $S_{4}$ & $m-\chi_{B}$ \\
\hline$m-\chi_{G}$ & $S_{4}$ & $m-\chi_{G}$ & $V_{G}$ & $V_{R}$ & $S_{4}$ \\
\hline$\mu_{E}$ & $m$ & m & $m-\chi_{G}$ & $\gamma$ & $\rho$ \\
\hline$\mu_{c}$ & $V_{B}$ & $\rho$ & $\mu_{c}$ & $S_{2}$ & $m$ \\
\hline$m$ & $\delta$ & $\mu_{c}$ & $m$ & $\mu_{E}$ & $\mu_{E}$ \\
\hline$\rho$ & $\mu_{E}$ & $\mu_{E}$ & $\rho$ & $\mu_{c}$ & $\mu_{c}$ \\
\hline$S_{2}$ & $\mu_{c}$ & $S_{2}$ & $S_{2}$ & $m$ & $S_{3}$ \\
\hline$m-\chi_{R}$ & $\sin 2 \chi$ & $S_{3}$ & $\sin 2 \chi$ & $\sin 2 \chi$ & $\gamma$ \\
\hline$V_{B}$ & $S_{2}$ & $\gamma$ & $S_{3}$ & $\rho$ & $S_{2}$ \\
\hline$S_{3}$ & $S_{3}$ & $V_{B}$ & $V_{B}$ & $m-\chi_{R}$ & $V_{B}$ \\
\hline $\sin 2 \chi$ & $V_{R}$ & $\sin 2 \chi$ & $m-\chi_{R}$ & $S_{3}$ & $\sigma_{\mathrm{R} \mathrm{H}}$ \\
\hline$\gamma$ & $m-\chi_{R}$ & $m-\chi_{R}$ & $\gamma$ & $V_{B}$ & $\sigma_{\mathrm{RV}}$ \\
\hline$\sigma_{\mathrm{RH}}$ & $\sigma_{\mathrm{RH}}$ & $\sigma_{\mathrm{RH}}$ & $\sigma_{\mathrm{RH}}$ & $\sigma_{\mathrm{RH}}$ & $m-\chi_{G}$ \\
\hline$\sigma_{\mathrm{RV}}$ & $\sigma_{\mathrm{RV}}$ & $\sigma_{\mathrm{RV}}$ & $\sigma_{\mathrm{RV}}$ & $\sigma_{\mathrm{RV}}$ & $\sin 2 \chi$ \\
\hline$\delta$ & $\delta$ & $\delta$ & $\delta$ & $\delta$ & $\delta$ \\
\hline
\end{tabular}

Class indices are 1: Open Water/Nilas (OW) 2: Young Ice (YI) 3: Smooth First Year Ice(SMFI) 4: Rough First Year/MultiYear Ice (RFMYI).

RADARSAT-2 Quad-Pol image, we employed a similar technique, which also consists of two steps: The first step comprises a feature extraction (a total of 18), the results of which are ingested into a neural-network-based classifier in the second step. Details of features used to classify the RADARSAT- 2 and classification accuracy data can be found in [8].

\section{Results AND Discussions}

The results of the proposed classifier can be seen in Figs. 4 and 6 (right-hand images). Next to these images, we display the $m-\chi$ RGB composite of the acquisitions for comparison, see Figs. 4 and 6 (left-hand images). In order to validate the stability of the training process, we randomly split the initial training data patches into two mutually exclusive subsets, i.e., training dataset and validation dataset. The classification results compared to validation data samples as presented in Table III exhibit a very promising accuracy, which underscores the stability of our algorithm. The percentages in the matrix indicate the proportion of samples of one reference class that were assigned to the respective ice type by the classifier. Therefore, columns add up to $100 \%$. The test was carried out for the RISAT-1 16:13:26 UTC acquisition (Fig. 4) with variations of less than $3 \%$ in the following accuracy matrix.

One can also detect a strong match between the visual structures in these $m-\chi$ RGB images and the pertaining classified images. Open water/nilas areas are clearly detected throughout both acquisition frames. Also the young ice portions are classified correctly except for a slight over representation in the far range. This is more likely due to a noticeable noise pattern (especially on the vertical margins) of the RISAT-1 18:13 UTC acquisitions. Such noise pattern related biases certainly need to be addressed when establishing the classifier (minimum $\sigma_{0}$ measurable by FRS- 1 imaging mode is $-17 \mathrm{~dB}$ ). Smooth first year ice portions, which appear as darker ice floes in the $m-\chi$ RGB image, are correctly detected, as well as the bright structures of more strongly deformed first year ice. Furthermore, a fairly reasonable degree of correspondence has been achieved between official ice chart and classified RISAT-1 CL-Pol imagery.

To arrive at a rigorous quantification of the information content of different CL-Pol features, we apply the concept of mutual information from information theory, which has become a rather central measure regarding the analysis of informational content and discriminative power. Intuitively, mutual information indicates the information overlap of two properties appearing in the same event, e.g., two polarimetric features or a polarimetric feature, and the ice class. For the former (two polarimetric features), a high value in mutual information indicates that both features carry the same information, i.e., have a high information theoretic correlation. When the mutual information of the ice class and a feature are higher (or lower) this implies a higher (or lower) class-predictive value of the respective feature. In order to increase the comparability we often use a normalization with the statistical entropy $\mathcal{H}$ of the respective property. For theoretical details, consult [8] and the references therein. In Table IV, one can infer the relative predictive strength of each feature with respect to the classification (in descending order). For Fig. 5, which indicates redundancy of features, we remark that the close relationship of $\mu_{E}$ and $\mu_{c}$ can be attributed to the mathematical fact that either of the features can be recovered from the other through a reversible function. $V_{G}$ and $m-\chi_{G}$ are fully interchangeable in their information content. Given that $H_{i}$ and $S_{1}$ differ merely by a log and some constants, this explains the third obvious redundancy in Fig. 5. These very obvious redundancies, all due to mathematical similarity of respective features, would allow us to discard three features in an operational classification toolchain. In light of these redundancies, it is also not surprising to find $H_{i}$ and $S_{1}$ in the same rank in Table IV, and to have identical or almost identical values for the pairs $\left(V_{G}, m-\chi_{G}\right)$ and $\left(\mu_{E}, \mu_{c}\right)$ in Table IV. For the other features, we conclude from Fig. 5 that any further analysis concerning CL-Pol-based classification is well-advised to not discard any of these features.

On the other hand, Table $\mathrm{V}$ shows the contribution of different features for a particular pair of class, which indicates that $H_{i}$ and $H_{p}$ provide significant information in order to discriminate open water and sea ice and also to characterize different types of sea ice. Given that $H_{i}$ and $S_{1}$ differ merely by a log and some constants, this explains the third obvious redundancy in Fig. 5. We also remark that the contribution of phase difference $(\delta)$ is rather limited for sea ice characterization.

In order to conform the reliability of the CL-Pol-based automated sea ice classification technique, we have compared a pair of near-coincident RISAT-1 FRS-1 (CL-Pol) and a RADARSAT-2 Fine Quad-Pol acquisition. As mentioned in the previous section, we adopted the methodology presented in [8] in order to classify the RS-2 imagery where an ANN classifier based on 18 polarimetric features have been proposed. Left-hand sides of Figs. 7 and 8 show $m-\chi$ RGB (RISAT- 

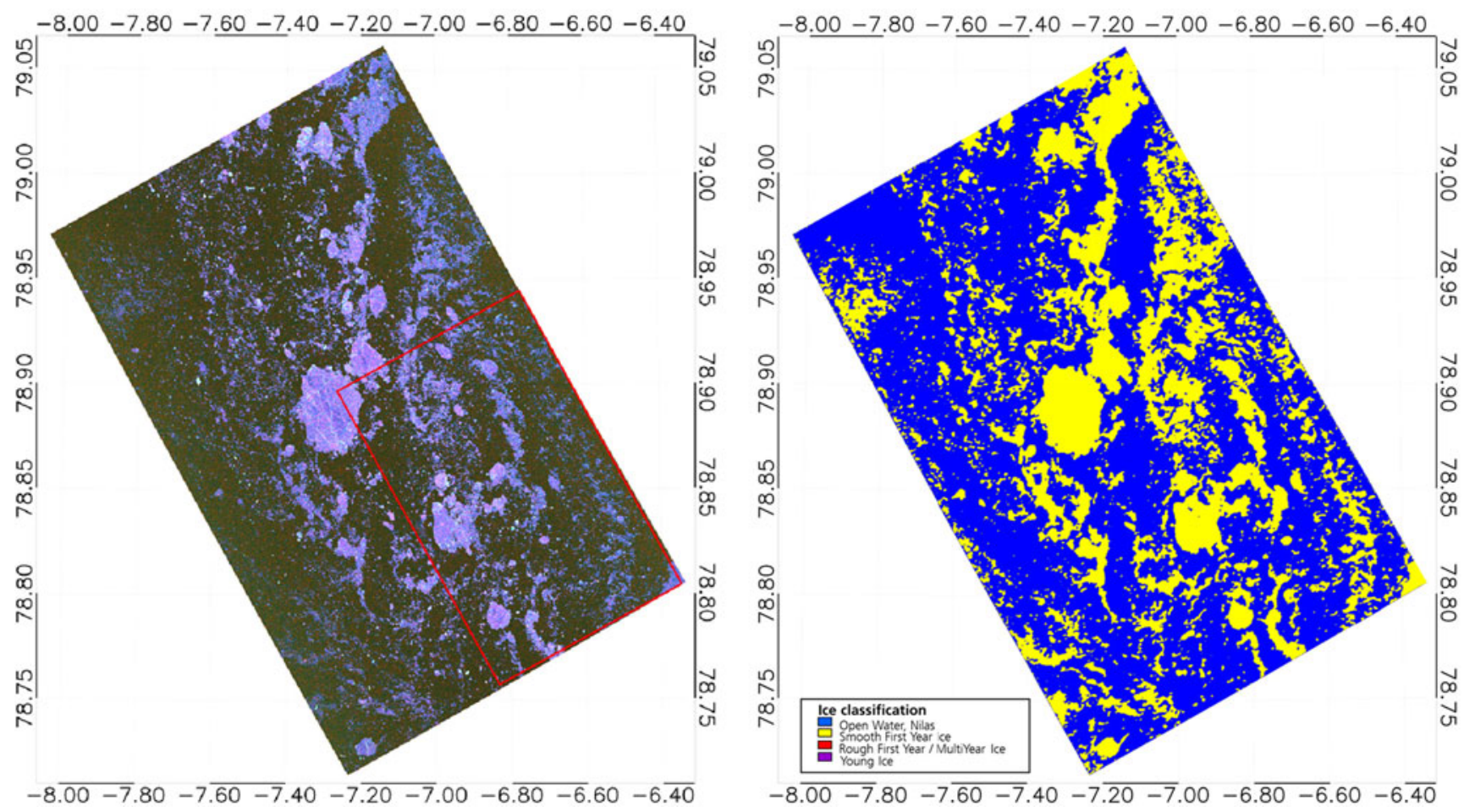

Fig. 7. Left: Geocoded $m-\chi$ RGB composite of RISAT-1 acquisition (FRS-1) on September 6, 2015, 16:38:29 UTC. Right: Classification result of RISAT-1 acquisition on September 6, 2015, 16:38:29 UTC. Open water/nilas (OW) blue, young ice (YI) purple, smooth first year ice (SFYI) yellow, rough first/multiyear ice (RFMYI) red.
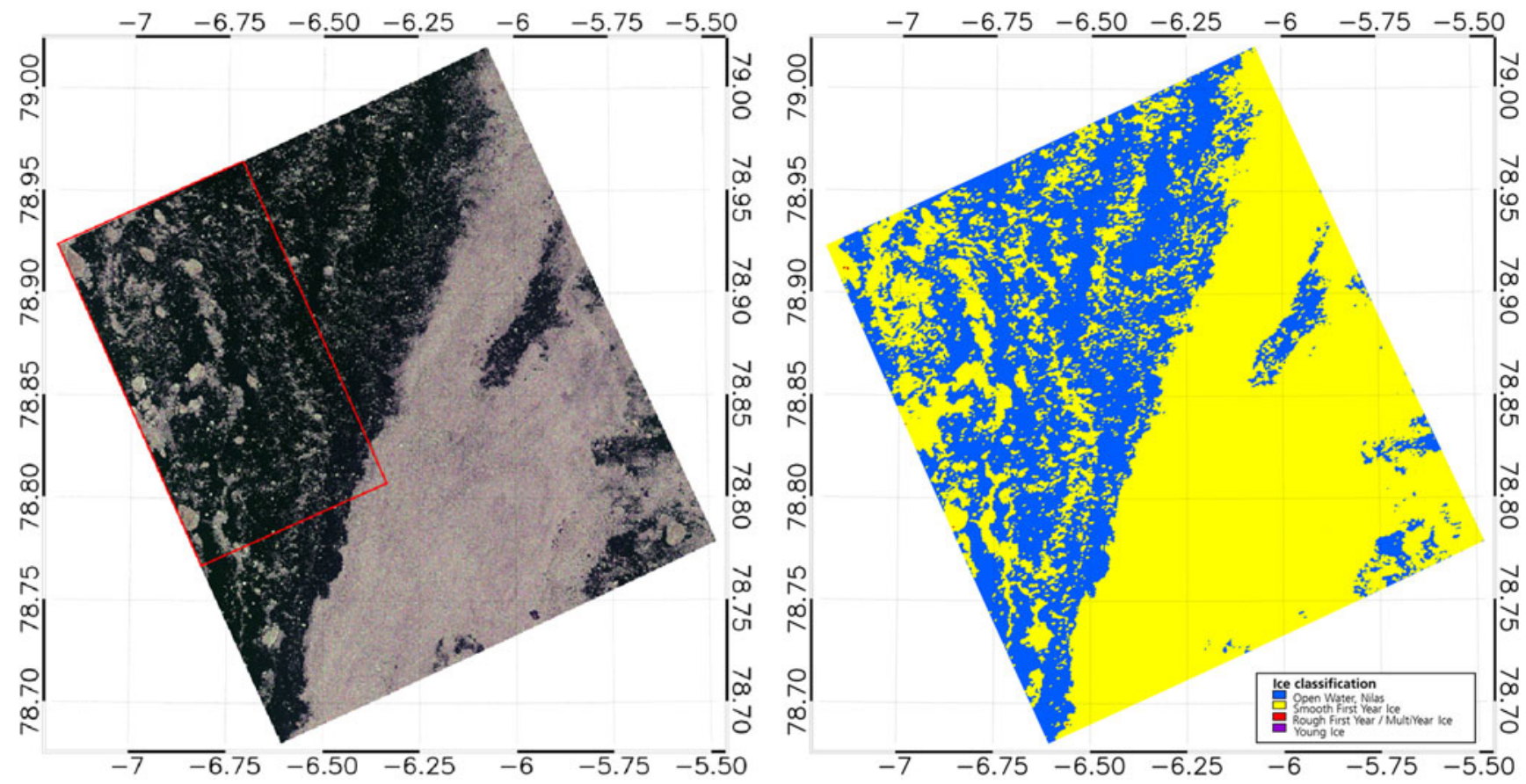

Fig. 8. Left: Pauli RGB composite image of RADARSAT-2 acquisition of September 6, 2015, 16:25 UTC. Right: Ice chart of RISAT-1 acquisition of September 6, 2015, bottom frame. Open water/nilas (OW) blue, young ice (YI) purple, smooth first year ice (SFYI) yellow, rough first/multiyear ice (RFMYI) red.

1) and Pauli RGB (RADARSAT-2) images and the spatial overlap is indicated with a red rectangle on both of the images. One can observe that in both of the images (mainly in spatially overlapped area) dominant ice classes are the same, i.e., Open water/nilas (OW) blue and smooth first year ice
(SFYI). Classification results obtained from this unique nearcoincident acquisition also correspond with visual observation and is presented in right-hand sides of Figs. 7 and 8. In case of RADARSAT-2 fully polarimetric data, an overall classification accuracy of $96 \%$ was reported in [8], which is similar to 
the overall classification accuracy achieved by using CL-Pol data (Table III).

\section{CONCLUSION}

We presented an algorithmic approach for automatically classifying ice types in CL-Pol SAR imagery. We used a CL-Pol dataset acquired through RISAT-1, the first satellite borne SAR system delivering CL-Pol products. Our process chain extracted polarimetric features and ingested the feature vectors in a neuralnetwork-based classifier. Classification results show promising quality when juxtaposed to feature images and also in terms of classification accuracy. Shannon Entropy (Polarimetric and Intensity component, i.e., $\left.H_{p}, H_{i}\right)$ and first stokes parameter, $S_{1}$, are found to be the most useful features in terms of CL-Polbased sea ice classification. A comparison was carried out on a near-coincident (spatially and temporally) CL-Pol and QuadPol (RADARSAT-2) $C$-band SAR image acquired over arctic sea ice with similar classification accuracy. After an extensive analysis and observations of present investigations, it is justified to conclude that CL-Pol SAR techniques have produced promising results, also when comparing with results from nearcoincident Quad-Pol acquisition. Future research will expand this approach to a wider array of CL-Pol datasets, possibly with simultaneous in situ data for validation, to explore the potential for operational purposes of sea ice classification.

\section{ACKNOWLEDGMENT}

RISAT-1 dataset was kindly provided by Kongsberg Satellite Services AS (KSAT), Troms $\varnothing$, Norway. The views, opinions, and findings contained in this paper are those of the authors and should not be construed as an official DLR position, policy, or decision.

\section{REFERENCES}

[1] A. V. Bogdanov, S. Sandven, O. M. Johannessen, V. Y. Alexandrov, and L. P. Bobylev, "Multisensor approach to automated classification of sea ice image data," IEEE Trans. Geosci. Remote Sens., vol. 43, no. 7, pp. 1648-1664, Jul. 2005.

[2] N. Y. Zakhvatkina, V. Alexandrov, O. M. Johannessen, S. Sandven, and I. Frolov, "Classification of sea ice types in ENVISAT synthetic aperture radar images," IEEE Trans. Geosci. Remote Sens., vol. 51, no. 5, pp. 2587-2600, May 2013.

[3] R. Ressel, A. Frost, and S. Lehner, "A neural network-based classification for sea ice types on x-band SAR images," IEEE J. Sel. Topics Appl. Earth Obs. Remote Sens., vol. 8, no. 7, pp. 3672-3680, Jul. 2015.

[4] R. Ressel, S. Singha, S. Lehner, A. Rösel, and G. Spreen, "Investigation into different polarimetric features for sea ice classification using x-band synthetic aperture radar," IEEE J. Sel. Topics Appl. Earth Obs. Remote Sens., vol. 9, no. 7, pp. 3131-3143, Jul. 2016.

[5] J. Karvonen, "Baltic sea ice concentration estimation based on c-band dual-polarized SAR data," IEEE Trans. Geosci. Remote Sens., vol. 52, no. 9, pp. 5558-5566, Sep. 2014.

[6] T. Geldsetzer and J. J. Yackel, "Sea ice type and open water discrimination using dual co-polarized c-band SAR," Can. J. Remote Sens., vol. 35, no. 1, pp. 73-84, 2009.

[7] M. A. N. Moen et al., "Comparison of feature based segmentation of full polarimetric SAR satellite sea ice images with manually drawn ice charts," Cryosphere, vol. 7, no. 6, pp. 1693-1705, 2013. [Online]. Available: http://www.the-cryosphere.net/7/1693/2013

[8] R. Ressel and S. Singha, "Comparing near coincident space borne C and $\mathrm{X}$ band fully polarimetric SAR data for arctic sea ice classification," Remote Sens., vol. 8, no. 3, p. 198, 2016. [Online]. Available: http://www.mdpi.com/2072-4292/8/3/198
[9] F. Charbonneau et al., "Compact polarimetry overview and applications assessment," Can. J. Remote Sens., vol. 36, no. sup2, pp. S298-S315, 2010.

[10] R. K. Raney, "Hybrid-polarity SAR architecture," IEEE Trans. Geosci. Remote Sens., vol. 45, no. 11, pp. 3397-3404, Nov. 2007.

[11] H. Li, W. Perrie, Y. He, S. Lehner, and S. Brusch, "Target detection on the ocean with the relative phase of compact polarimetry SAR," IEEE Trans. Geosci. Remote Sens., vol. 51, no. 6, pp. 3299-3305, Jun. 2013.

[12] M. Dabboor and T. Geldsetzer, "Towards sea ice classification using simulated RADARSAT Constellation Mission compact polarimetric SAR imagery," Remote Sens. Environ., vol. 140, pp. 189-195, 2014. [Online]. Available: http://www.sciencedirect.com/science/article/pii/ S003442h5713002939

[13] M. M. Espeseth, C. Brekke, and S. N. Anfinsen, "Hybrid-polarity and reconstruction methods for sea ice with 1- and c-band SAR," IEEE Geosci. Remote Sens. Lett., vol. 13, no. 3, pp. 467-471, Mar. 2016.

[14] A. A. Thompson, "Overview of the radarsat constellation mission," Can. J. Remote Sens., vol. 41, no. 5, pp. 401-407, 2015.

[15] M. Arkett et al. "Preparation by the canadian ice service for the operational use of the RADARSAT constellation mission in their ice and oil spill monitoring programs," Can. J. Remote Sens., vol. 41, no. 5, pp. 380-389, 2015.

[16] H. Li and W. Perrie, "Sea ice characterization and classification using hybrid polarimetry SAR," IEEE J. Sel. Topics Appl. Earth Obs. Remote Sens., vol. 9, no. 11, pp. 4998-5010, Nov. 2016.

[17] S. Nissen, "Neural networks made simple," Software 2.0, 2005. [Online]. Available: http://fann.sourceforge.net/fann_en.pdf

[18] R. K. Raney, J. T. Cahill, G. Patterson, and D. B. J. Bussey, "The m-chi decomposition of hybrid dual-polarimetric radar data with application to lunar craters," J. Geophys. Res., Planets, vol. 117, 2012, Art. no. E12.

[19] R. Shirvany, M. Chabert, and J.-Y. Tourneret, "Estimation of the degree of polarization for hybrid/compact and linear dual-pol SAR intensity images: Principles and applications," IEEE Trans. Geosci. Remote Sens., vol. 51, no. 1, pp. 539-551, Jan. 2013.

[20] T. Geldsetzer, M. Arkett, T. Zagon, F. Charbonneau, J. J. Yackel, and R. K. Scharien, "All-season compact-polarimetry c-band SAR observations of sea ice," Can. J. Remote Sens., vol. 41, no. 5, pp. 485-504, 2015.

[21] L. T. Pedersen, M. B. Kreiner, and B. Hackett, "Product user manual: For regional high resolution sea ice charts greenland region," Tech. Rep. CMEMS-OSI-PUM-011-003, 2015. [Online]. Available: http://resources.myocean.eu/

[22] S. R. Cloude, D. G. Goodenough, and H. Chen, "Compact decomposition theory," IEEE Geosci. Remote Sens. Lett.,, vol. 9, no. 1, pp. 28-32, Jan. 2012.

[23] IDL. Interactive data language description, 2017. [Online]. Available: http://www.harrisgeospatial.com/ProductsandSolutions/Geospatial Products/IDL.aspx

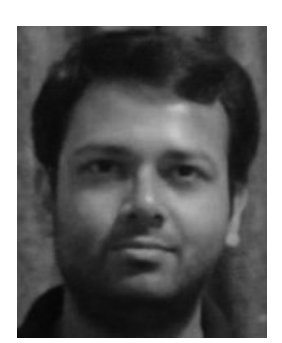

Suman Singha (M'15) received the M.Tech. degree in remote sensing from the Indian Institute of Technology, Roorkee, India, in 2009, and the M.Sc. degree in remote sensing and the $\mathrm{Ph} . \mathrm{D}$. degree in microwave remote sensing from the University of Hull, Hull, U.K., in 2010 and 2014, respectively.

He was a Visiting Scientist with the European Maritime Safety Agency, Lisbon, Portugal, from March to July 2012. Since 2013, he has been with Maritime Safety and Security Laboratory, German Aerospace Center, Bremen, Germany, as a Research Scientist. His research interests include the application of artificial intelligence and machine learning approaches to classify traditional and polarimetric SAR images with emphasis on near real-time maritime applications.

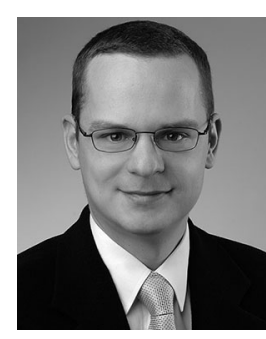

Rudolf Ressel received the Diploma degree in mathematics from Georg-August-University, Göttingen, Germany, in 2008, and the Ph.D. degree in mathematics from the University of Bremen, Germany, in 2012 , in the field of inverse problems.

In July 2013, he joined the German Aerospace Center, where he works as Research Scientist with Maritime Safety and Security Laboratory, Bremen. His research interest are ice type classification by means of SAR image analysis. 